Our aim was to assess whether routine screening for TV in females is indicated in an urban Australian setting.

Methods Females attending a sexual health clinic from July 2013-February 2014 who were tested for Chlamydia trachomatis (CT) and Neisseria gonorrhoeae (NG) were eligible to have a TV test on the same specimen. Testing was performed by transcription-mediated amplification on female genital specimens using the Aptima Trichomonas vaginalis assay (Hologic Inc., United States). Characteristics of the study population were examined.

Results During the study period, 393 women were tested for CT/NG on 471 occasions. TV tests were performed 347 (73.7\%) of CT/NG specimens. There were no significant differences between women who had $(\mathrm{n}=294)$, and did not have ( $\mathrm{n}$ = 99), a TV test during the study period, except that women who had recent overseas sexual contact were less likely to be tested. Of the 347 tests, two TV infections were diagnosed, a positivity rate of $0.6 \%$ (95\% CI $0.07-2.1 \%)$. Both cases were Australian-born with a history of injecting drug use in the past 12 months, Neither were sex workers and one identified as Aboriginal. One presented with post-coital bleeding, and TV was identified on wet film. The other reported pelvic symptoms, but was tested on outreach and no wet film microscopy was performed. Neither had concurrent CT/NG infections detected.

Conclusion We found a low positivity rate of TV among female attendees. Both TV infections were in women who had symptoms suggestive of a sexually transmitted infection. Our findings are in accord with those from previous urban Australian studies and do not support routine TV screening for asymptomatic women in metropolitan Sydney.

Disclosure of interest statement Aptima Trichomonas vaginalis assay testing kits were provided free by Hologic (Australia) Pty Ltd.

\section{P12.07 DELIVERING INCREASED SERVICE PROVISION: IPPF EXPERIENCE OF PROVIDING TARGETED TECHNICAL SUPPORT WITHIN EXISTING SRH SERVICE DELIVERY POINTS FOR STRENGTHENED STI-SPECIFIC SERVICE PROVISION}

${ }^{1} \mathrm{D}$ McCartney*, ${ }^{2} \mathrm{D}$ Andjelic, ${ }^{3} \mathrm{~A}$ Singh. ${ }^{1}$ International Planned Parenthood Federation, London, UK; ${ }^{2}$ IPPF Western Hemisphere Region, New York, USA; ${ }^{3}$ IPPF South Asia Region, Delhi, India

\subsection{6/sextrans-2015-052270.487}

Introduction In 2013, the International Planned Parenthood Federation (IPPF) set out a strategy for increasing service provision across the IPPF Member Associations (MAs). A key service area identified for rapid scale-up was sexually transmitted infections (STIs). As a global service provider of sexual and reproductive health (SRH), the prevention and management of STIs has long been an integral part of IPPF's mission. However, the provision of services for STIs, other than HIV, has been a lower priority among many IPPF MAs in recent years.

Methods To support the strengthening of STI-specific services, small seed grants were provided to eight MAs covering all six of IPPF's geographical regions to conduct a rapid assessment of existing service delivery points. This action-oriented process enabled MAs to set their own goals and directions, and to prioritise interventions to address STIs through existing service delivery points. Specific assessments determined the current service capacity and identified technical assistance needs for increasing STI-specific service delivery.
Results The assessments of existing service delivery points gathered information on key issues affecting access, utilisation, and quality of care services for STIs, and identified staff training needs. Overall analysis led to the identification of specific deficiencies affecting service delivery and need for improving programme-level interventions including training and updating guidelines for STIs. Some key opportunities included the scalingup of both syndromic management and etiological diagnosis in all service delivery points, strengthened integration with other SRH-related services, rollout of rapid diagnostic syphilis testing, and prioritising interventions for young people including vaccination, prevention and screening.

Conclusion With commitment and support, targeted technical assistance with limited resources enabled the development of strategic recommendations for scaling-up effective STI-specific services. A number of existing simple, effective, and cost-effective services were identified for implementation towards increasing quality STI services at existing service delivery points providing SRH services.

Disclosure of interest statement Nothing to declare.

\section{P12.08 STI MANAGEMENT IS HIV PREVENTION: IMPROVING ACCESS TO A COMPREHENSIVE PACKAGE OF STIGMA- FREE SRH AND HIV SERVICES FOR KEY POPULATIONS BY IMPLEMENTING THE LATEST WHO GUIDELINES}

${ }^{1} \mathrm{D}$ McCartney* ${ }^{*},{ }^{1} \mathrm{~T}$ El Hajj, ${ }^{2} \mathrm{D}$ Bakomeza, ${ }^{3} \mathrm{~N}$ Jagdish, ${ }^{4} \mathrm{D}$ Maloti, ${ }^{5} \mathrm{~A}$ Tena, ${ }^{6} \mathrm{~S}$ Sokolowski. ${ }^{1}$ International Planned Parenthood Federation (IPPF); ${ }^{2}$ Reproductive Health Uganda; ${ }^{3}$ Family Planning Association of India; ${ }^{4}$ Family Health Options Kenya; ${ }^{5}$ Cameroon National Family Welfare Association; ${ }^{6}$ German BACKUP Initiative, GIZ

\subsection{6/sextrans-2015-052270.488}

Introduction "Shadows and Light" is a three-year project implemented by four IPPF Member Associations and funded by the German BACKUP Initiative. The project developed service capacity that addressed the linked sexual and reproductive health (SRH) and HIV needs of four key populations: transgender people (India); sex workers (Uganda); people who use drugs (Kenya); and men who have sex with men (Cameroon). Often at increased risk of STIs, screening, diagnosis and treatment of STIs are crucial parts of a comprehensive response to HIV.

Methods The initial activities focused on preparing clinic sites, including training of service providers to provide stigma-free services. This involved consultations with key population networks and peer educators to inform development of a full continuum of HIV services, including other STIs, as part of SRH services. Recommended interventions were guided by available WHO guidelines for key populations, and included an assessment of implementation in line with current recommendations.

Results The project contributed to the development of stigmafree SRH services that offered safe access for key populations in each clinic site. The assessment of available STI services found that while syndromic management for all key populations was available, there was limited availability of targeted screening for asymptomatic STIs. While serological testing for syphilis infection was available in some sites, none were screening for gonorrhoeal or chlamydial infections. No periodic presumptive treatment for asymptomatic STIs was undertaken.

Conclusion The inclusion of SRH-related recommendations in the WHO consolidated HIV guidelines for key populations were a critical advancement. By creating a strong link that STI management is HIV prevention enables a greater possibility of addressing within programmes funded by the Global Fund to 